\title{
Tourisme sportif et construction de l'altérité dans les espaces de pratique : le canoë en France et en Zambie
}

Antoine Marsac

\section{(2) OpenEdition}

Journals

Édition électronique

URL : http://journals.openedition.org/tourisme/190

DOI : 10.4000/tourisme. 190

ISSN : 2492-7503

Éditeur

Éditions touristiques européennes

\section{Édition imprimée}

Date de publication : 1 juin 2013

Pagination : 84-97

ISSN : 2109-5671

\section{Référence électronique}

Antoine Marsac, «Tourisme sportif et construction de l'altérité dans les espaces de pratique : le canoë en France et en Zambie », Mondes du Tourisme [En ligne], 7 | 2013, mis en ligne le 30 septembre 2015, consulté le 01 mai 2019. URL : http://journals.openedition.org/tourisme/190 ; DOI : 10.4000/ tourisme.190

\section{(c) (i) $\odot$

Mondes du tourisme est mis à disposition selon les termes de la licence Creative Commons Attribution - Pas d'Utilisation Commerciale - Pas de Modification 4.0 International. 


\section{Tourisme sportif et construction de l'altérité}

\section{dans les espaces de pratique :}

\section{le canoë en France et en Zambie}

Antolne Marsac

[antoine.marsac@u-bourgogne.fr]

Maître de conférences en sociologie, Laboratoire SPMS- EA 4I80, Université de Bourgogne

Résumé. Historiquement, le canoë s'est constitué autour du principe de libre circulation sur les cours d'eau et sur un imaginaire touristique de la nature. Dans la France de l'après-guerre, des parties de rivières se sont fermées à la navigation, conduisant les canoéistes à rechercher de nouveaux espaces de pratique. Des sites d'eau vive ont alors été identifiés dans les pays africains durant les années 1950. Une logique "naturelle", fondée sur un environnement instable et sauvage, s'est constituée selon des conceptions touristiques de la nature. Ces dernières prennent en compte le rapport au territoire et le rapport à l'Autre. II convient de saisir comment a émergé, du point de vue des touristes occidentaux, leur relation aux populations Tonga de Zambie, dont une partie est mobilisée comme guides lors des descentes du Zambèze en canoë. Des entretiens menés avec eux ont été analysés à partir d'une enquête in situ, dans la pratique itinérante. Cette activité est destinée à faire découvrir la nature et à explorer des hauts lieux. Les gorges du Zambèze en aval des chutes Victoria constituent en ce sens un site propice à ce type d'activité lors des voyages en Zambie. Mais dans quel contexte ce loisir s'est-il diffusé d'Europe en Afrique ? II s'agit de montrer comment les rapports d'altérité entre pratiquants se sont construits et comment ils changent les normes des guides.

Abstract. Historically, canoeing is based on the principle of free circulation on water streams and on a tourism-oriented overview of nature. In France, during post-war years, rivers were closed to navigation, which led canoeists to search for new spaces to practice. Wild waters sites were identified in African countries during the 1950s. A "natural" logic, based on an unstable and wild environment, has been incorporated under tourist designs of nature. These take into account the relationship with the land and the relation to the Other. We should understand how emerged, from the point of view of Western tourists, their relationship to the Tonga people of Zambia, some of them being mobilized as guides on the Zambezi River. Interviews were analyzed, using an in situ investigation. This activity is designed to explore the nature and explore high places while travelling in Zambia; the Zambezi River Gorge is a world-renowned site for this type of activity. In what context did leisure canoe spread from Europe to Africa? We should show how the otherness between practitioners relationships are constructed and how they change the guides norms. 
À la fois sport de compétition, loisir et moyen de déplacement dans les pays du Sud, le canoë est depuis des décennies une pratique présente sur tous les continents. En se proposant d'analyser ses conditions d'émergence et de développement en France puis en Zambie, nous questionnons sa mise en tourisme dans les territoires français et zambien. Comment ce loisir s'est-il diffusé en Occident et quel est son retentissement pour les groupes locaux en Zambie ?

Pour répondre à ces interrogations premières, deux études, l'une historique et l'autre ethnographique, ont été menées sur l'évolution de la composition sociale des guides de canoë dans des territoires touristiques. La première repose sur un travail d'analyse des archives du Canoë Club de France (CCF), association créée à Paris en 1904, dont la vocation est "l'encouragement au tourisme nautique ${ }^{(1)}$ ". La seconde s'est déroulée en 2006 à partir d'une observation participante menée avec des guides africains. Aux côtés de ces canoéistes locaux, des guides européens (français et britanniques) s'adonnent à la descente de rivière dans le cadre de leur travail de professionnels du tourisme. Le tourisme sportif est défini ici par l'organisation d'activités et la visite d'un territoire, à partir desquelles se déroule le séjour.

L'enquête ethnographique a consisté à observer les guides de tourisme sportif et leurs clients en Afrique australe en avril 2006 dans la vallée du Zambèze. Ce haut lieu jouit d'une renommée mondiale dans le secteur du tourisme sportif car ce cours d'eau est le quatrième fleuve africain. Il sépare le Zimbabwe de la Zambie. Le second cité est un État d'Afrique australe enclavé peuplé d'un peu plus de dix millions d'habitants. Il est composé de groupes bantous divisés en chefferies jusqu'à l'arrivée des Européens au XIX ${ }^{e}$ siècle. Puis, le pays est colonisé par les Britanniques de 1924 à 1964 sous le nom de Rhodésie du Nord et devient un lieu de villégiature pour la bourgeoisie sudafricaine. S'il s'urbanise depuis les années 1930 (Hannerz, 1983), son économie est aujourd'hui tournée principalement vers l'agriculture et l'extraction de minerais. Depuis trente ans, le tourisme se développe également dans les grandes villes (Livingstone et Lusaka, la capitale). Des infrastructures hôtelières y ont été aménagées par les Britanniques pour accueillir les touristes étrangers et leur proposer des descentes en canoë. Le lieu d'enquête, Livingstone, est la "capitale touristique" du pays, située à cinq kilomètres des chutes Victoria, l'un des sites les plus visités du monde. En période estivale, cette localité double sa population. Mais, le reste de l'année, la raison saisonnière s'efface pour laisser place à une cohabitation entre étrangers. Nous sommes donc parti de ce questionnement : qu'est-ce qui caractérise l'altérité dans le domaine du tourisme sportif en France et en Zambie, tout en considérant qu'il s'agit de deux moments historiques différents ? Le dessein de cet article est de caractériser les relations sociales liées aux pratiques de tourisme sportif, c'est-à-dire d'activités auxquelles s'adonnent les touristes encadrés par des guides professionnels locaux. Nous définissons la notion d'altérité comme ce qui caractérise le lien avec l'Autre et rend compte d'une dimension inconnue (Lévinas, 200I).

À partir de cette perspective, l'on interroge la diffusion du canoë de rivière par la mise en tourisme des territoires et les relations entre acteurs (Eichberg, 1998). Il s'agit de saisir comment ont émergé les interactions sociales entre Africains et Français résidant en Zambie. Nous avons observé des canoéistes des entreprises spécialisées ayant leur siège près des gorges du Zambèze dans le site des chutes Victoria (à la hauteur du pont frontière entre la Zambie et le Zimbabwe). L'enquête se focalise sur l'organisation du canoë comme forme d'excursion destinée à découvrir la nature ${ }^{(2)}$ et à rechercher des sites propices à l'exploration. Dans quelle conjoncture cette activité de loisir s'est-elle diffusée d'Europe en Afrique ? Comment les relations d'altérité entre pratiquants de canoë et riverains se construisent-elles ?

Cette étude conduit à opérer un retour socio-historique sur les conditions d'émergence des relations entre touristes et populations locales. Nous avons choisi de nous centrer sur le tourisme sportif en canoë plutôt que sur les pratiques communément plébiscitées par les prati- 
quants occidentaux (safari, rafting...) car la tradition du trip, séjour de tourisme sportif, renvoie à l'exploration du territoire, propre aux canoéistes. Il s'agit également de s'appuyer sur une démarche ethnographique pour tenter de saisir l'émergence de la relation à l'autre, c'est-à-dire à celui qui se distingue, à ces périodes et dans ces contextes, de l'homme occidental dans le cadre des descentes en canoë. En considérant les changements induits par les apports de la commercialisation de ce loisir en Zambie depuis la décolonisation, on s'aperçoit qu'une enquête ethnographique s'avère nécessaire pour saisir les relations entre les pratiquants des activités dites de "découverte du milieu naturel" et les Tonga, groupe ethno-linguistique dont sont issus les guides. Un retour par une analyse sociohistorique pose ici des jalons pour comprendre l'évolution du loisir dans les rivières depuis son introduction en Zambie jusqu'à son développement au sein des communautés britannique et française présentes dans ce pays.

\section{MÉTHOdologie}

Comprendre l'imbrication du phénomène d'altérité implique de situer notre étude à la fois dans une ethnographie des canoéistes mais aussi dans une histoire des pratiques. Il existe un enjeu heuristique à investiguer empiriquement les relations entre guides africains et européens car cette analyse est le fruit d'une étude articulant les concepts de deux disciplines des sciences sociales: l'anthropologie et l'histoire. Les présupposés théoriques et méthodologiques se focalisent sur des questions propres aux approches comparatives. Ce recours à deux disciplines pose des problèmes épistémologiques (du fait des exigences méthodologiques que ces approches requièrent) car anthropologues et historiens ne partagent pas les mêmes critères de scientificité (Blanchard, 1995) ni les mêmes modes d'administration de la preuve (Passeron, Boutier et Revel, 2006). Il faut donc préciser que l'articulation entre ces disciplines interroge un même objet à des niveaux de réalité différents (Izard et Bonte, 1991) et s'y limite. L'historicité se combine à l'étude in situ des groupes s'adonnant au tourisme. L'altérité est ici interrogée grâce à cette double perspective. La recherche historique a été conduite par analyse de contenus de la revue La Rivière ${ }^{(3)}$ et de sources rassemblées dans une sélection d'ouvrages spécialisés ${ }^{(4)}$ traitant des relations des premiers touristes résidant avec les riverains.

La première démarche consiste à s'appuyer sur une définition théorique de l'altérité. D’après Lévinas (200I), plusieurs acceptions de ce terme coexistent. L'altérité renvoie nécessairement aux relations avec les Autres, que nous bornons ici aux populations locales. Le travail procède, en complément des archives, d'une analyse de données menée par entretiens répétés, réalisés avec des acteurs ayant structuré le champ du canoë. Interrogés sur leur manière d'appréhender l'étranger (Leite et Graburn, 20 I0), des guides ont répondu aux questions que nous leur avons posées lors d'entretiens. Nous avons délimité l'altérité, au sens où celle-ci repose sur des éléments subjectifs (imaginaires, symboliques). Les discours des touristes interrogés représentent des sources complémentaires à l'analyse historique centrée sur l'écrit. Les entretiens semi-directifs consistent à recueillir directement ces discours d'acteurs par des questions préalablement formulées. Un dialogue s'établit entre le chercheur et son informateur. Le premier pose des questions précises au second, à partir d'un dispositif nommé guide d'entretien. En fonction des réponses, chacun ajoute des éléments significatifs dans le guide d'entretien. Il s'agissait ici d'interroger les guides zambiens sur leurs relations entretenues avec les touristes occidentaux et sur leur "cohabitation" au sein du territoire.

Ensuite, nous avons procédé à une comparaison entre ces deux populations. La dimension comparative a été développée comme dans les travaux d'histoire conduits entre l'Allemagne et la France (Caritey et Krüger, 2007). Les auteurs comparent des manifestations gymniques ayant lieu dans deux contextes nationaux différents. Nous pouvons rapprocher notre démarche de cette étude qui s'attache à analyser la construction de l'altérité comme "figure de comparaison". Les apports historiques sur lesquels repose ce travail s'inspirent des analyses de Caritey 
et Krüger, qui replacent la question de la diffusion des sports pour saisir la structuration progressive des espaces de pratique. Notre étude mobilise le modèle des processus longs en sociologie (Elias, 1991). L'analyse des récits permet de comprendre l'évolution des usages du fleuve par les canoéistes pour les replacer dans leur contexte d'émergence. En complément, l'intérêt du regard ethnographique réside dans l'étude des facteurs culturels et des normes sociales. Le traitement des données a été réalisé via une analyse thématique qui repose sur un modèle explicatif des actions et des représentations. Le matériau recueilli nous permet de présenter des résultats traduisant les dynamiques d'altérité.

\section{LITTÉRATURE}

Cette recherche s'inscrit dans le domaine des études touristiques car elle s'attache à montrer l'origine des interactions et des clivages de représentations des populations. Dans le sillage des travaux historiques, de nombreuses analyses ont retracé la genèse des imaginaires des acteurs. Les travaux sur l'histoire du tourisme (Boyer, 1996) ont introduit la question des rapports du citadin à l'espace naturel et sa relation aux autres. Une liberté nouvelle au xxe siècle s'exprime dans les provinces (Bertho Lavenir, 1999). Viard revient sur ces sensibilités des citadins à la nature (Viard, 1990). Dressant une partition entre campagne, ville et nature, celui-ci sou- tient que la nature est une notion urbaine. Les citadins y perçoivent un intérêt qui, outre la lecture du paysage, promeut la rencontre avec l'Autre (Williams, 1977). Opposée en apparence à l'exploitation productive (culte du "naturel"), cette forme d'aménagement du paysage participe profondément de la même philosophie urbaine (maîtrise de la nature, rationalité spatiale). Le romantisme anglo-saxon du XVIII ${ }^{e}$ siècle marque un tournant en créant un univers débarrassé des traces du travail productif, sans référence aux producteurs (Chamborédon, 1980). L'invention du paysage croise ainsi la problématique de l'exploitation des espaces naturels dans le cadre des pratiques touristiques. Il s'agit d'une sensibilité à la nature qui s'apparente à un mimétisme des goûts des classes dominantes, sans référence aux populations paysannes. La bourgeoisie britannique s'abreuve de promenades hors des villes, et cherche à conserver les espaces naturels comme les forêts puis les cours d'eau propices au canotage. La nature explorée se dissocie des fonctions agraires que lui assigne le paysan. Si le culturalisme insiste sur l'invention de la nature (Williams, 1977), il convient de s'appuyer sur ces lectures pour comprendre l'émergence des rapports tissés entre touristes et locaux. Pour ce faire, nous partons de l'œuvre de Halbwachs (1970 [ ['rédition 1938]) consacrée aux besoins de retour à la nature (Boudes, 20I I). Pour rendre compte des interactions entre touristes et guides, il convient d'appré- hender ces influences du traitement de la nature et du rapport d'altérité. De fait, la littérature spécifique insiste sur un système de normes appréhendé à travers des usages de l'espace et les retentissements lors des périodes coloniales en Afrique (Blanchard, 1995). En ce sens, il faut revenir à l'histoire de la Zambie pour comprendre comment le canoë s'est établi par le code de l'indigénat et l'exploration des espaces naturels (Decraene et Châtel, 1995, p. 77), car des excursionnistes ont préfiguré au $\mathrm{XX}^{\mathrm{e}}$ siècle l'avènement d'usages des territoires étrangers.

\section{EXCURSIONNISME}

ET EXPLORATION DE

\section{LA NATURE EN CANOË}

Nous devons d'abord décrire la diffusion des normes sur lesquelles reposent les sensibilités à la nature en fonction des périodes d'exploration du Canoë Club dans la première moitié du XX siècle, en France puis en Rodhésie du Nord, pour comprendre les pratiques contemporaines des guides de tourisme à l'origine des relations avec les touristes occidentaux.

Le canoë a longtemps été considéré par les nobles comme une forme de résurgence d'une pratique ancienne révélée à la faveur de la société victorienne et de l'Empire. Une élite britannique va investir des territoires inconnus grâce à son temps libéré. À la fin du XIX siècle, l'Écossais MacGrégor, venu en France en 1867 pour naviguer en "autonomie" sur les fleuves, annon- 
çait les prémices de la rencontre avec les riverains. Rétablis dans des dimensions diachroniques, ces faits entrent dans la problématique de la construction de l'altérité en canoë. Introduit en France dans un contexte d'exploration des vallées rurales, le canoë ne s'est institutionnalisé qu'en 1904 avec la création du CCF. L'apparition de cette pratique est liée à la batellerie et aux usages des voies navigables ayant transformé le canoë, jadis utilitaire, en un loisir distinctif et bourgeois. Jusqu'aux années 1930, l'activité est fondée sur un idéal de libre exploration de la nature. En effet, le CCF privilégie l'exploration des torrents jugés "sauvages” par ses adeptes. L'écriture de récits de croisière met en évidence un modèle de tourisme à la croisée du tourisme et du sport ${ }^{(5)}$. Les auteurs indiquent à leurs lecteurs les points de vue sur la rivière avec les chemins d'accès. On y retrouve les normes de "l'excursionnisme cultivé", termes qui renvoient aux qualités particulières d'une activité et de ses adeptes, telles qu'elles apparaissent dans cette description du canoéiste: "Mystérieux à souhait, il a ce regard lointain que donne l'examen des problèmes ardus et l'habitude des responsabilités, il connaît les cartes et les rivières par cour. Conquérant des eaux vierges, il fend avec son étrave la vigueur des torrents inconnus. Une mission sacrée l'appelle vers des pays creusés de canyons sans soleil où vivent des indigènes datant de l'enfance $d u$ monde $^{(6)}$." Cet extrait démontre que, dans le rituel d'écriture aux- quels s'adonnent les membres du CCF, l'altérité et la nature occupent la place centrale.

L'intrusion dans l'espace de la littérature de sensibilités à la nature liées à l'exploration et à la domination des citadins sur les populations rurales rappelle que nous nous situons en période coloniale. Le terme "indigènes", utilisé pour désigner les habitants, est devenu une catégorie de perception de l'autre. Il apparaît dès les premières années du Canoë Club dans l'évocation de ces "contrés inconnues peuplées

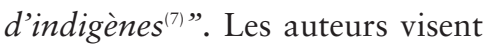
ceux qui ne possèdent pas les modes de mobilité dont jouissent les canoéistes, en majorité parisiens. Le vocable "indigènes" lié à la domination urbaine se généralise dans les récits de croisière. Bien que la terminologie permettant de qualifier les populations locales ait évolué au fil du temps, il s'agit d'une extension du terme signifiant ici "habitants des vallées”. Jusqu'aux années 1940, les discours des promoteurs de l'excursion restent marqués par un certain paternalisme envers les populations rencontrées. Le marquage social de l'excursionnisme s'enracine dans une sensibilité à la différence de l'autre. Il subsiste dans les récits une partition entre "eux" et "nous". Cette désignation des populations constitue un marqueur de l'appartenance sociale et est, par conséquent, l'élément à prendre en compte dans l'altérité. Les archives de la revue La Rivière décrivent des populations rurales rencontrées au gré des descentes de cours d'eau.
Le canoë constitue en cela un révélateur des lignes de fracture entre les promoteurs d'une exploration des espaces "vierges" et la pratique des compétiteurs qui apparaît au $\mathrm{XX}^{\mathrm{e}}$ siècle. Deux raisons expliquent ce clivage. D'abord, le récit des "premières”, sur le modèle de l'alpinisme, met en avant une nature "immaculée" peuplée "d'indigènes". Ensuite, la mission "civilisatrice" des excursionnistes dans les provinces françaises préfigure les expéditions lointaines. Les sensibilités des membres du club se diffusent dans des publications portant sur le tourisme : imaginaire du retour à la terre, conquête du pays et liens avec l'autre.

Si l'expression "canoë-kayak" n’apparaît qu'en 1949 dans les publications des topoguides Susse et de la revue Camping et plein air, elle s'introduit aussi dans l'industrie des loisirs comme en témoigne son usage dans l'entre-deux-guerres (Halbwachs, 1970 [ I re édition 1938], p. 175). La plasticité des besoins identifiée par cet auteur il y a plus de soixante-dix ans peut être rapprochée de la situation contemporaine. En effet, l'excursionnisme repose sur un besoin d'évasion, des visées pédagogistes et fonde un imaginaire des grands espaces hérité de la culture anglaise, des épopées canadiennes et des explorations de sociétés savantes. En France, cette diffusion est placée sous le signe du "réveil des provinces" (La Soudière, 2008, p. 89). Les représentations qu'ont les guides français de la naturalité incluent les représentations 
de ces territoires ruraux. Ce groupe met en œuvre une rationalisation de l'exploration des sites. Le développement de la pratique repose ainsi sur une construction précise de l'altérité. Pour les auteurs des récits du Canoë Club, "l'indigène" fait partie intégrante de la nature. Ce contexte influence les visées exploratrices des membres du CCF, créant de nouveaux besoins.

\section{L’ALTÉRITÉ “UTILE” : CANOÉISTES VS “INDIGÈNES"}

Dès la création du club, les membres du CCF se sont forgé un rapport touristique au cours d'eau. En prônant des idéologies héritées de l'hygiénisme, les adhérents parisiens de l'association ont cherché à rompre avec leurs conditions de vie quotidiennes. Étudiant spécifiquement cette période, Catherine Bertho Lavenir dissocie les croisières en eau calme des descentes de rivière d'eau vive. Pour qualifier les rivières parcourues par les membres du CCF, elle utilise les termes de "cours d'ean accidentés, propres au loisir", qu'elle oppose aux "itinéraires touristiques" des croisières, promenades "sans aucun danger réel" (Bertho Lavenir, 1999, p. 30I). Ce club orienté vers le tourisme cherche à se distinguer des autres sociétés nautiques (de voile ou d'aviron) en prônant la découverte de l'autre dans un torrent perçu par ses membres comme "vierge", sans se limiter aux voies navigables (fleuves, canaux). Ses membres fondent une éthique liée à la lenteur du séjour touristique qui se démarque de la sensibilité de l'époque exprimée par une recherche toujours accrue de vitesse.

Le CCF organise des croisières en groupe, des cours, des conférences sur la faune, la flore des cours d'eau agrémentés de séances photo. Ce club fut membre fondateur dans les années trente de l'Union nationale des associations de tourisme, qui contribue à développer les descentes en canoë. Des récits de croisières archivés au club relatent les exploits de ses membres et rendent compte de leur manière de percevoir les autres et les provinces dans des descriptions naturalistes et romancées. La représentation des torrents comme espace de jeu semble être communément partagée. Les canoéistes, attachés à l'exploration "sauvage" de la nature, bénéficient ici d'une légitimité parmi les cercles mondains de la capitale. La figure de "l'indigène" y occupe une place centrale car elle est immortalisée par les récits. Dans ces descriptions, l'aventure est synonyme de découverte de nouveaux paysages, de rencontres avec les "indigènes". Le terme "indigènes" est ici usité au pluriel car il indique autant un rapport au territoire exploré, au voyage effectué loin de la civilisation et au contact d'inconnus, qu'un rapport aux populations qu'il s'agit de questionner dans le but de renseigner les membres du CCF : "Il y a eu de nombreux interviews que nous dûmes avoir nous-mêmes avec les indigènes, interviews souvent contradictoires... et parfois dangereux" (récit de croisière du CCF publié dans La Rivière). Ces "indigènes" communiquent aux auteurs des informations "utiles" sur la région touristique dans laquelle coule le cours d'eau, la superficie de son bassin versant, la longueur parcourue de sa source au confluent et son débit. Cette sensibilité à la nature liée à la domination des Parisiens sur ces populations rurales rappelle que les élites jouissent d'une mobilité et possèdent une culture héritée des sociétés savantes de la capitale (le CCF sera primé à la Société de géographie en 1929). Ainsi, le terme “indigènes”, usité pour désigner les riverains des vallées qui contribuent, grâce à leur aide, à l'exploration, au déroulement de l'excursion, se généralise dans La Rivière.

Si des formes plurielles de sensibilité à la nature ont pu éclore au CCF, c'est qu'il existe des tensions à propos de la définition de la pratique, entre le tourisme et la compétition. Il y a cette propension des élites membres du CCF à rechercher une nature peu peuplée pour la modeler en cherchant à développer les pratiques entre sport, opportunités et idéologie de l'“escapisme ${ }^{(8) "}$. À la "pureté de la nature" s'opposerait la "dégradation des conditions de vie urbaine" qui est parfois associée à celle de la compétition - que dénoncent les membres du club jusqu'à la Seconde Guerre mondiale. Si l'activité de loisirs transforme la naturalité des torrents, la Fédération française de canoë s'y intéresse. Elle est créée en 1932 pour rassembler les clubs, former des compétiteurs et favoriser le développement tou- 
ristique des cours d'eau. Ses membres œuvrent pour les expéditions en réalisant des films et en publiant des récits de voyage. Le guide sera la figure de proue de l'institution bien que la vocation touristique de cette fédération passe au second plan à mesure que se développent les compétitions de canoë.

À partir de la moitié du XX siècle, la descente touristique des torrents associée à la "vie sauvage"(9) est relayée par la propagande du tourisme par le Canoë Club. Dès lors, l'activité renvoie aux représentations des espaces dits "vierges". En répondant à la question posée par le rapport des pratiquants au milieu naturel, on avancera que l'exploration des rivières s'est transformée par l'influence de mécanismes sociaux de perception de l'espace dit "sauvage" à partir de l'héritage des excursionnistes du CCF. La pratique du canoë dans les torrents renvoie à un imaginaire des grands espaces inscrits dans l'histoire du continent américain et des grandes épopées. Cet espace de représentations pousse des Occidentaux à entreprendre des périples de plusieurs mois dans des pays aux "antipodes de la vieille Europe” (Nil, Zambèze). En fait, les expéditions lointaines ont succédé aux croisières en France ou outre-mer. Une frange de pratiquants refusant les aménagements des rives se tourne vers les voyages lointains comme les descentes du Zambèze. Le contexte des années 1950 est à la libre circulation sur ces cours d'eau fondée sur un ima- ginaire de la nature, sur le modèle du canoeing et des épopées. L'ethos de "conquête" des membres du Canoë Club se déploie à travers cette opération symbolique de fuite vers la nature, qui puise sa légitimité dans la détestation de ce que ses membres, pour la plupart notables, nomment "la civilisation des villes $^{(10)}$ ". Ces derniers instaurent une norme de mobilité au sein du CCF rendue possible grâce au progrès des modes de déplacement en train et à la villégiature. Il s'agit d'explorer la nature par le voyage et d'écrire un récit des descentes réalisées en totale autonomie sur plusieurs jours.

En 1938, Halbwachs décrivait en ces termes ce contexte touristique: "Des groupes toujours plus nombreux, une partie croissante de la population a pris ainsi le goût des beaux horizons, des séjours en des cités pittoresques, étrangères, exotiques, des sports, l'habitude aussi des cures prolongées en des endroits salubres, auprès de sources bienfaisantes. Curiosité, amour des arts et de la nature, des exercices et performances physiques, 'plaisir d'arriver tard en un sauvage lieu', goût des voyages? Mais il y a là tout un ensemble d'habitudes, d'exigences, qui n'ont pas toujours existé, en tous cas à ce degré [...]. Rendons grâce à l'industrie hôtelière, touristique, à l'industrie des transports, chemins de fer, bateaux, automobiles, à la réclame, à la publicité, qui sont aussi des industries, à la presse, aux imprimeurs de guides, de récits de voyages, aux fabricants de canoës, de kayaks, de skis, de tout le matériel et des costumes sportifs [...] : c'est à eux que nous devons tous ces besoins, et non pas seulement la possibilité de les satisfaire" (Halbwachs, 1970 [I 're édition 1938]).

Cependant, cet engouement des membres du CCF dure jusqu'au milieu des années 1950 car le réseau hydrographique s'amenuise (deux cents barrages sont édifiés pour les besoins d'électrification de la France de l'entre-deux-guerres). Les torrents des croisières s'assèchent. En 1954, la majorité des rivières en état est descendue. À la manière des expéditions des alpinistes, le Canoë Club propose de découvrir les rivières étrangères. "La rivière doit couler dans une nature sauvage, à l'accès difficile, où la civilisation a peu pénétré et où l'indigène est resté à l'état primitif. Des enseignements précieux doivent être rapportés (rivière, climat, végétation, faune, peuplade ${ }^{(11)}$." Nous montrerons, dans la partie suivante, comment cette incursion dans les torrents lointains a prolongé l'exploration au Canoë Club.

\section{LE FLEUVE AFRICAIN : UN TERRITOIRE PROPICE AUX DESCENTES DE RIVIÈRE}

L'exploration des territoires à des fins touristiques conduit à analyser les conditions de pratique des premiers guides travaillant dans le canoë. En effet, ces professionnels du secteur touristique cherchent à "capter" les fractions aisées des 
Occidentaux (Cousin et Réau, 2009).

À partir de la fin des années 1950, les explorateurs promeuvent l'itinérance au long cours dans des torrents africains. Cette démarche, renforcée par l'allongement de la durée des congés, constitue une alternative aux croisières dans les cours d'eau les plus touchés par la sécheresse. Pour comprendre l'engagement vers des voyages en Rhodésie du Nord, il paraît indispensable d'en connaître la structure et les changements à travers l'histoire de ce territoire. Les membres du CCF partent pour l'Afrique australe, sur le Zambèze, en 1950, à la hauteur des chutes Victoria. Le Zambèze, dans ce pôle d'attractivité touristique, fait partie de ces hauts lieux qui jouissent également d'une renommée mondiale. Dès son indépendance, en 1964 , l'État zambien manifeste son intérêt pour l'activité physique, d'abord en fonction de priorités sanitaires puis dans le cadre du développement touristique. Cette jeune nation présente des régions à fort contraste de développement ${ }^{(12) .}$ La province du sud, où sont situées Livingstone et les chutes Victoria, est l'une des moins pauvres avec Lusaka, capitale administrative, plus prospère. L'activité de canoë y est particulièrement développée. Des infrastructures y ont été aménagées pour accueillir les touristes qui contemplent les chutes Victoria en leur proposant des descentes dans les eaux plus calmes du fleuve. Pour le voyageur pressé, il s'agit d'une activité essentiellement dévolue aux Occidentaux, permettant d'agré- menter leur séjour. Mais dans la mesure où l'activité est organisée par des guides locaux ${ }^{(13)}$, des interactions plus poussées peuvent se créer entre canoéistes et Zambiens. Lorsque ce territoire correspondait à l'ancienne Rhodésie du Nord, des guides organisant le séjour y ont été formés par les Anglais pour accueillir les touristes. Madoré, ancien directeur de la Fédération française de canoë-kayak, revient sur cette exploitation des torrents comme une tradition du CCF relayée par une propagande au travers des films réalisés, une "tradition de plein air”. Pour lui, les canoéistes promeuvent une forme d'exotisme dans l'activité à travers la découverte de nouveaux territoires comme le Zambèze : "Les pratiques d'aprèsguerre à l'époque du CCF, c'était les classes dominantes qui se sont regroupées dans les années 19501960 au Grand Rex avec des photos, des premières. [...] Ils s'étaient regroupés en sociétés savantes et fédéraient les loisirs d'eau vive en canoë. Ils ont utilisé le chemin de fer. Cette tradition de plein air s'est interrompue dans les années $1970^{(14)}$." Ces guides ont développé l'organisation d'expéditions, perfectionné les techniques d'approche du cours d'eau et fait évoluer le matériel de canoë.

Le marché de l'outdoor, terme recouvrant ces activités en milieu naturel, est particulièrement représenté dans le secteur du tourisme sportif. On y retrouve des tour-opérateurs détenteurs d'une licence d'agent de voyages, qui s'appuient sur l'attractivité locale dans le secteur du tourisme. Au-delà des formules de courts séjours, les sociétés proposent des trips durant lesquels les clients issus des pays occidentaux d'Europe - Anglais, Français, Allemands -, d'Amérique du Nord ou d'Afrique du Sud s'adonnent au canoë (Mounet et Chifflet, 1996), guidés par des Zambiens. Les destinations des pays du Sud sont aussi recherchées comme alternative à la surfréquentation des vallées françaises (comme dans l'Ardèche). Sur le Zambèze, la découverte des cultures locales (notamment de l'ethnie Tonga, vivant près de la frontière entre Zambie et Zimbabwe, au nord des chutes Victoria) est aujourd'hui la première raison qui pousse les jeunes pratiquants à voyager. La composition de la population canoéiste constitue un indicateur précis dans la manière d'appréhender l'altérité. Le caractère élitiste de ces pratiquants et l'hégémonie $\mathrm{du}$ citadin sur les riverains $\mathrm{du}$ Zambèze marquent ces séjours.

Mais comment cette passion du canoë va-t-elle cristalliser les relations entre Occidentaux et Africains? Les deux groupes se retrouvent propulsés dans des interactions qui favorisent des débats sur les façons d'organiser l'activité, les manières de naviguer, l'utilisation du matériel. Cette destination revient à de très nombreuses reprises dans les entretiens comme la référence en matière de trips pour les canoéistes occidentaux, au même titre que l'Everest pour les alpinistes. Pour les touristes se rendant en 
Zambie, il ne s'agit plus seulement d'allier vacances et dépaysement. Dans leurs discours, le séjour occupe une place centrale. Dans cette perspective, le voyage devient un moment d'émotions partagées, vécu avec les populations locales, les Tonga, sur un mode intense, au prisme de la différence culturelle (Wieviorka, 2008). Mais il faut s'écarter de ce constat sommaire pour se prémunir des fausses interprétations et des jugements péremptoires. Une description du cas zambien soulève des questions quant aux modes de relations entre individus des pays du Nord et individus des pays du Sud. Quelle est la forme d'altérité marquée dans le cas du tourisme sportif ? Quels sont les enjeux sociaux sous-tendus lors des descentes du Zambèze en canoë ?

En Afrique, les canoéistes français trouvent des lieux propices à l'exploration des espaces naturels au contact des populations du Zambèze : "Une sorte de retour aux origines de l'activité, dans lequel chacun redécouvre les joies et la rusticité de la rivière : l'angoisse qui saisit le canoéiste au moment d'embarquer, la reconnaissance des difficultés aux abords des passages délicats avec les locaux"(entretien avec un canoéiste français, 2006). La quête d'une villégiature de qualité (camps au bord de l'eau, ambiance des berges, liens avec les riverains) et la recherche d'un entre-soi sont parmi les raisons pour lesquelles ces canoéistes parcourent des milliers de kilomètres pour explorer le torrent. À l'instar des travaux traitant de l'attractivité des sites touristiques (Gagnon, 2007), étudions aussi les nouvelles formes d'exploration aventureuse en identifiant la ressource territoriale sur laquelle se réalisent ces pratiques liées aux représentations de l'espace local.

\section{DES PRODUITS D’AVENTURE ANCRÉS DANS \\ LE TERRITOIRE ZAMBIEN}

Il s'agit maintenant de décrire les modes de diffusion de l'offre en analysant l'organisation de l'aventure en Zambie. Pour les guides locaux, ils encadrent les touristes dans les eaux tumultueuses de "leur" fleuve. Or pour les clients, la pratique est choisie et non utilitaire, car son déroulement s'inscrit dans une temporalité calquée sur le modèle libéral de la prestation de services. Ces canoéistes consomment un trip d'une semaine sur le Zambèze. Le malentendu avec les guides zambiens est à ce titre éloquent. Ces pagayeurs naviguent depuis plus de vingt ans sur le fleuve. Les guides travaillant sur ce territoire pointent même une désaffectation des sites français au profit des destinations étrangères. À ce titre, le Zambèze est devenu un "haut lieu" du tourisme sportif comme en témoignent les produits proposés par les tour-opérateurs, dans lesquels l'exotisme domine. La figure du guide perçu comme nonchalant et désinvolte est une réminiscence de la condition de "l'indigène" dans le secteur du tourisme. Dans les brochures, les Africains sont mon- trés dans une posture accueillante, confinés dans leur communauté. L'exploration en canoë s'inscrirait dans cette recherche de communion avec l'Autre. Le contact entre populations et la découverte de paysages des sites visités transcenderaient le voyage. La réciprocité du lien est ici soulignée: "Ce contact avec les touristes, ça a ouvert l'esprit des guides locaux" (entretien avec un touriste français).

La dimension d'exploration possède des résonances post-colonialistes car l'altérité s'apparente aux idéologies de la conquête du monde (Denis et Pociello, 2000). Cette activité perçue comme vertueuse par ses promoteurs comporte une teneur utilitariste en arrière-plan. Le canoë autorise ce rappel des symboles du colon. La navigation sur les rivières rejoue en quelque sorte l'expansionnisme des empires coloniaux. Elle s'est constituée autour des pratiques et dans l'imaginaire collectif de l'aventure. Des lodges pour les groupes sont aménagés dans les sites. Cet élargissement des lieux de tourisme demeure inséparable des transformations de l'espace conditionné par la mise en place d'un réseau d'acteurs (guides, agences de voyages) qui diffusent des produits d'aventure. Alors qu'une partie des jeunes du pays s'occidentalisent, leur rapport au fleuve demeure dicté par des préceptes religieux (communion avec les esprits). Les Tonga organisent des cérémonies en faveur des esprits du fleuve, rite perpétué sur les rives du Zambèze. Dans le même temps, le cours d'eau perd 
les fonctions séculières qui lui étaient assignées (transport, lavage des hommes et des animaux) au profit de la production électrique ${ }^{(15)}$ et de l'essor des produits d'aventure. Or le tourisme sportif s'enracine dans le processus de différenciation des groupes en Zambie. Ces mutations laissent supposer que le pays a connu une évolution rapide par une autonomie symbolique (Bayart, 2006). Dans ce contexte, le tourisme perturberait la culture Tonga de tradition orale.

Mais l'aventure se transforme sous l'influence de la culture occidentale. D'une part, la vocation de ces descentes en canoë passe de l'excursionnisme à l'ethnotourisme et au sport dit "extrême"; ces nouveaux loisirs sportifs s'enracinent dans le défi lancé à l'hédonisme. D'autre part, l'émergence de nouvelles territorialités induit une adaptation des touristes aux courants des fleuves du monde entier. On peut émettre l'hypothèse que ce changement est dû à la globalisation des produits touristiques (Robertson, 1992). Les guides africains seraient enclins à vendre d'autres prestations pour se distinguer de l'offre locale. En témoignent les descentes du Zambèze en rafting et les safaris, pratiques aventurières réinscrites dans l'offre des tour-opérateurs. Mais le dégoût suscité par le tourisme sportif chez une partie des Zambiens se manifeste, voire se théâtralise, lors de manifestations comme la promotion des produits d'aventure par les tour-opérateurs. Les guides entrent en conflits avec les riverains à cause de la mise en scène des coutumes Tonga. Ces tensions révèlent les clivages avec les acteurs locaux. La commercialisation des produits de canoë distingue les guides, qui maîtrisent l'usage technique et les enjeux économiques, des Tonga qui entretiennent d'autres rapports à la nature. En Zambie, le tourisme s'articule autour de deux pôles : la visite des parcs naturels et le tourisme sportif promu par des citadins. Le rapport urbain à la pratique locale est questionné par les catégories de la globalisation culturelle (Bayart, 2006). La globalisation désigne ici les caractéristiques de la période qui succède à la décolonisation. Elle est un facteur d' "internalisation" sur la scène de relations interpersonnelles, qui est le domaine d'exercice de toute investigation ethnologique (Bazin et Selim, 2002, p. 303). Cela renvoie tant aux différentes pratiques qu'aux comportements afférents tels que les manières d'accoster, de se parler dans l'accès au site d'embarquement, l'engagement dans une pratique risquée ${ }^{(16)}$. L'adoption de modes de se tenir se répercute sur la construction de l'altérité dans la mesure où le global imprime sa marque sur la conception légitime des pratiques de ces étrangers. Lors des trips, on retrouve la même relation de condescendance de la part des guides européens qui jugent leur manière de pratiquer l'activité comme étant la seule légitime ${ }^{(17)}$. Les touristes se rendant en Zambie se munissent de cartes géographiques de la région traversée. Cela conduit les promo- teurs de l'activité à adopter une position ethnocentrique. Car l'effort pour un Zambien de s'inscrire comme acteur est constant et les clivages que nous avons pu mettre en évidence à travers le développement des descentes commerciales de canoë ne sont pas nouveaux mais leur perpétuation au sein de la population constitue une forme de ségrégation.

Sur les rives du Zambèze, le localisme des Tonga prévaut. L'ethnographie se déplaçant dans les communautés zambiennes, l'observation in situ permet d'analyser les interactions entre touristes et locaux. Pour ces derniers, l'assujettissement à des choix qui ne sont pas personnels est lourdement connoté négativement. Ces Zambiens reprochent aux touristes de polluer les sites, de détériorer les rives ou encore d'exclure les pêcheurs et de favoriser la spéculation immobilière. Le canoë, à travers le tourisme dans les parcs naturels, se réalise dans le cadre d'activités liées à des situations de grande permissivité. Les canoéistes sont considérés par les Tonga comme des symboles du colon du fait de la pauvreté des populations exclues des profits engendrés par le tourisme ${ }^{(18)}$. Les pratiquants occidentaux distinguent les ethnotouristes des guides en leur attribuant des traits caractéristiques (attributs physiques, comportements types). La construction de l'altérité devient une dimension "classante" pour les clients des trips. Pour l'interpréter, analysons les catégories d'altérité comme un facteur central du déve- 
loppement du tourisme lié aux effets de la globalisation.

\section{L’ALTÉRITÉ : DES CONFLITS AUX EFFETS DE LA GLOBALISATION}

La situation contemporaine peut être maintenant ré-appréhendée à partir d'une lecture par le conflit. Le recours au concept de globalisation pour interpréter les problèmes persistants d'altérité qui se traduisent par des conflits nous conduit à analyser cette relation complexe entre le rapport à l'Autre et au territoire hôte. Partant de ce contexte, on peut se demander dans quelle mesure la globalisation culturelle influe sur le secteur du tourisme sportif.

D'un côté, les réactions des groupes locaux au processus de globalisation s'inscrivent dans un système de relations sociales et d'interdépendances où la contrainte collective fait que toute conduite hérétique contraire aux normes dominantes du tourisme sportif est disqualifiée. Mais la majorité de la population zambienne demeure spectatrice de la commercialisation de cette activité à laquelle les touristes s'adonnent. Depuis la décolonisation, la communauté cède la place à la société, la tradition à la rationalité stratégique déterminée par les contraintes d'un système libéral. Ce développement profite donc partiellement à la population zambienne.

D'un autre côté, que ce soit dans le tourisme sportif ou dans le sec- teur minier, l'expérience du travail des Tonga, au lieu d'enrichir, consacre la discrimination ethnique. Ce qui aujourd'hui fonde le "désir d'ailleurs" (Michel, 2004) se pose dans les interactions déséquilibrées entre touristes et guides locaux, car c'est toujours la culture occidentale qui domine dans la relation à l'Autre (Latouche, 1989). C'est elle qui fixe ses normes, y compris lorsque les locaux s'approprient la pratique. Le canoë demeure une activité marquée par les normes occidentales. Certes, elle renvoie à l'imaginaire des grands espaces mais l'activité provient de la naissance du besoin de s'évader des villes par l'exotisme et de découvrir une population aujourd'hui dominée par l'économie libérale et survivant près des rives du fleuve.

Le vocabulaire utilisé par les tour-opérateurs en Zambie présente des similitudes avec la rhétorique des explorateurs du CCF. La mise en scène des coutumes locales serait vécue comme un moment charnière dans les liens tissés entre Zambiens et Occidentaux. Les Tonga jusquelà rétifs à l'emploi de pseudonymes se sont convertis à cette manière de se nommer sous l'effet du tourisme. En Zambie, le partage d'un ordre global avec les guides locaux se heurte à des réticences dans la mesure où les règles du canoë sont celles de quelques guides promoteurs qui appartiennent aux classes dominantes. Comme les premiers guides de rivière sont des Occidentaux, les infrastructures du tourisme sportif zambien liées aux tour-opérateurs reproduisent les fonctions de diffusion de ces sports lancés par les Britanniques et les Français. Mais la relation d'altérité demeure confinée dans un déséquilibre social et culturel entre populations, à cause notamment des inégalités en termes de richesse. Marc Augé a rapproché récemment la démarche de l'ethnologue de celle du touriste: "Ils appartiennent tous les deux à la partie du monde la plus favorisée, celle qui est capable d'organiser des voyages d'agrément ou des voyages d'étude chez les Autres" (Augé, 2009, pp. 58-59). Mais la comparaison s'arrête là. L'ethnologie repose sur une forme d'objectivation du rôle de l'enquêteur dans la production de connaissance. Augé poursuit d'ailleurs en ce sens : "Il n'y aurait aucun scandale si tous les hommes pouvaient être touristes ou ethnologues, si la mobilité des uns n'était pas un luxe alors que la mobilité des Autres est un destin ou une fatalité" (ibid.). En adoptant son point de vue, on pourrait conclure à une relation en sens unique de l'Occident vers les pays du Sud. L'opposition utilité-inutilité du canoë structure les discours autour de l'hégémonie occidentale caractérisée par les formes de capacité à être mobile. À la mobilité subie s'opposerait la mobilité choisie. C'est dans cette dernière que s'inscrit la séquence des touristes en partance pour un trip en Zambie. Ses conditions présentent les capacités des touristes à s'affranchir d'une existence qu'ils perçoivent comme "aseptisée". Dans le cas de 
l'aventure, le client compose avec la dimension du dépaysement opposé au localisme de sorte que l'altérité s'entremêle dans l'expérience globale des guides.

De même, Marc Boyer démonte le déséquilibre de la relation entre l'ethnotourisme et les populations locales à travers le fantasme du "sauvage" et sa survivance. Il décrit ce qu'il nomme "le contact avec les groupes humains sauvages". Il montre que les "ethnotouristes veulent voir ces peuples dont l'allure, les coutumes, les pratiques sont objet à la fois d'attirance et de répulsion" (Boyer, 1996, p. 25). Les jeunes pour qui c'est le premier voyage en Afrique accolent ce terme "sauvage" issu d'un ensemble de représentations autour du mythe des rivières "sauvages" et du wilderness aux populations Tonga. La figure de "l'indigène" est revivifiée par les nécessités commerciales de l'ethnotourisme et de l'aventure. Ce modèle de tourisme légitime une culture touristique dans le sport. Le tourisme apparaît paternaliste et dominateur (Chamborédon, 1980) car il émane des conceptions de la bourgeoisie cultivée et d'une idéologie du développement des vallées françaises. Les fractions de classes aisées des canoéistes iront jusqu'à parler, à l'instar des exploits des alpinistes, "de conquête du torrent" en qualifiant le fleuve et sa population riveraine. Parmi ces citadins, beaucoup s'exilent vers une vie en contact avec les Zambiens et le milieu naturel puis deviennent guides à leur tour. Leur démarche consiste à créer des liens avec d'autres guides fréquentés dans les vallées et les villes. Les guides, en raison de leur expérience, reçoivent cet apprentissage de l'altérité pour apprendre à se situer dans la hiérarchie sociale, notamment dans leurs rapports avec les citadins. De tels rapports d'acculturation postcoloniale et leur extension dans des sociétés locales posent le problème de la cohabitation de groupes avec les valeurs dominantes. Ce sont de telles dynamiques qui se dégagent de la mise en scène des rapports entre guides et clients. Ce mouvement n'existe qu'en relation avec l'expérience des inégalités et la profonde domination qu'exerce le tourisme sportif dans une démarche consumériste: "Derrière la figure emblématique du touriste, pratique bouc émissaire ou icône intouchable, c'est l'être humain tout court qui se montre souvent ignorant ou inquiétant, ou les deux" (Michel, 2004, p. 486). Le statut d'un touriste le conduit nécessairement à endosser des rôles variés faisant émerger cette multiplicité des matrices identitaires (Goffman, 1961). Le tourisme sportif favorise des formes d'agrégation sociale autour d'une pluralité observée des processus d'identification à l'autre, au groupe et à l'activité. La fonction identitaire assignée au territoire renvoie à l'appartenance des ethnotouristes à une "communauté éphémère”. Le voyage s'inscrit dans une dimension identitaire du groupe liée à ces effets de la globalisation.

\section{CONCLUSION}

Si l'on s'intéresse aux origines de la pratique du canoë en France et en Afrique, on s'aperçoit que le développement de cette activité a partie lié avec les anciennes sociétés coloniales. Ainsi les loisirs de rivière en Zambie se sont considérablement diversifiés et recouvrent aujourd'hui des usages normés. Des conflits, des adaptations, des rénovations ont traversé la pratique légitime du canoë. Puis, le rafting et le safari, bien qu'assez anciens, sont apparus il y a trente ans dans ce pays à la faveur de l'utilisation d'engins de loisir par les Occidentaux. Nous validons donc l'hypothèse de l'impact de la globalisation sur les relations touristiques, valorisant l'exploration des territoires sur lesquels s'organise le tourisme sportif. L'activité est liée aux représentations de l'espace global. Mais des processus s'entremêlent. La relation qu'instaurent les acteurs détermine l'éclosion de conflits locaux avec les riverains.

La déterritorialisation des codes du tourisme sportif s'impose aujourd'hui comme l'une des caractéristiques du monde occidental contemporain. Elle est aussi l'une des principales forces de cet univers mondialisé car elle permet, dans le cas de populations déplacées, de susciter une distance face à la culture d'origine. Néanmoins, la globalisation culturelle nourrit l'idéologie libérale qui consiste à mettre en scène une partie de la population zambienne tout en l'exploitant. Aussi, le séjour présente les carac- 
téristiques de l'utopie : il s'agit d'un instant vécu dans un espace où vit l'Autre par l'exploration d'une nature urbanisée. Animés par l'idéal de commercialiser des produits d'aventure, les guides traduisent un réenchantement du monde dans le sens où ils éloignent les touristes des conflits et de la relation déséquilibrée qui s'instaure entre les tour-opérateurs et les locaux. Le partage avec les Européens pourrait être perçu comme un moment de convivialité. Or, en définitive, la démarche des touristes repose sur un "ethos de conquête" fondé sur des rapports d'altérité qui s'enracinent dans ce phénomène global d'industrie touristique en Afrique et qui tendent à exclure les plus pauvres.

\section{NOTES}

(I) En tête de la revue La Rivière, 1924.

(2) Le caractère naturel du torrent est une notion difficile à définir. L'enjeu de ce travail est d'en cerner les propriétés pour les touristes. Aussi, il faut rester prudent et retenir : ce qui a été peu modifié par l'homme.

(3) Publication parue de 1924 à 1974. Elle succède au Bulletin mensuel du Canoë Club, paru de 1906 à 1924

(4) Albert MAHUZIER, Le Livre d'or des Mahuzier, Presses de la Cité, 1980 ; Id., Quarante ans d'aventure, Presses de la Cité, 1971 ; Dr Georges GaUberT, Canoë quand même!, éditions Havas, 1950. Sources complémentaires aux archives du CCF.

(5) Cela signifie qu'il existe un tourisme "sportif" (le terme n'apparaît qu'à partir des années 1980) et un "excursionnisme" dont les formes varient mais qui cohabitent par- fois sur les mêmes sites de pratique du canoë.

(6) La Rivière, numéro spécial de 1943 en hommage à Albert Glandaz, p. 5.

(7) "Sur le Loir" (rubrique "Carnet du canoéiste"), Bulletin mensuel du Canoë Club, $n^{\circ} 51,1910$.

(8) Mouvement de rejet de la ville.

(9) La Rivière, numéro spécial de 1954, p. 66.

(I0) Extrait d'un récit de croisière, 1935.

(I I) La Rivière, numéro spécial de 1954 p. 67.

(I2) Une enquête de l'Agence française de développement (2012) montre un faible revenu du pays en dépit d'une aide publique au développement assez conséquente, utilisée notamment pour investir dans le secteur touristique.

(I3) Ce terme est employé en référence aux notions usitées par les acteurs. II signifie ici "populations locales".

(14) Entretien avec Hervé Madoré, inspecteur principal de la Jeunesse et des Sports, à Paris, le 15 février 2005

(I5) Située dans la région de la Copperbelt, principal site économique du pays.

(16) Le canoë zambien enregistre une accidentologie nulle par rapport aux accidents de saut à l'élastique et de rafting recensés en 2012 par la direction du Tourisme de Zambie (bureaux de Lusaka et de Livingstone)

(17) Ayant suivi quatre kayakistes et les safety kayakers (chargés de la sécurité) ayant participé à un "trip" sur le Zambèze, nous nous sommes imprégné de la mise en scène touristique de la pratique au contact des guides.

( 8 ) Le revenu médian par personne majeure ( 15 ans et plus) est de 50000 kwachas, soit à peine 20 \$US par jour.

\section{RÉFÉRENCES BIBLIOGRAPHIQUES}

Marc AugÉ, Pour une anthropologie de la mobilité, Seuil, 2009.

Laurent BAZIN et Monique SÉLIM,

"Ethnographie, culture et globalisation.

Problématisations anthropologiques du marché", Joumal des anthropologues, $n^{\circ} 88$ -

89, 2002.

Jean-François BAYART, L'État en Afrique. La politique du ventre, Fayard, 2006.

Catherine BerTHo LAVENIR, La Roue et le stylo. Comment nous sommes devenus touristes, Odile Jacob, 1999.

Pascal Blanchard. L'Autre et Nous. "Scènes et types" : anthropologues et historiens devant les représentations des populations colonisées, des "ethnies", des "tribus" et des "races" depuis les conquêtes coloniales, Syros, 1995. Marc BOYER, L'Invention du tourisme,

“Découvertes”, Gallimard, 1996.

Philippe BOudEs, "Morphologie sociale et sociologie de l'environnement : l'apport de Halbwachs à l'étude des relations entre les sociétés et leur milieu naturel', L'Année sociologique, vol. 61, $n^{\circ}$ I, 201 I.

Benoît CariteY et Michael Krüger, "Les fêtes nationales de gymnastique en Allemagne et en France (1860-1914)", dans André Gounot, Denis Jallat et Benoît CARITEY, Les Politiques au stade, étude comparée des manifestations sportives du XIX siècle au $X X^{e}$ siècle, Presses universitaires de Rennes, 2007.

Jean-Claude Chamborédon, "Les usages urbains de l'espace rural : du moyen de production au lieu de récréation", Revue française de sociologie, vol. 21, n I, 1980.

Saskia Cousin et Bertrand RÉAu, Sociologie du tourisme, La Découverte, 2009. 
Philippe Decraene et Bénédicte ChÂTeL, Onze leçons sur l'Afrique australe, CHEAM, 1995.

\section{Daniel DeNIS et Christian Pociello,}

À l'école de l'aventure : pratiques sportives de plein air et idéologie de la conquête du monde (1890-1940), Presses universitaires du sport, 2000

Henning EICHBERG, Body Culture. Essays on Sport, Space and Identity, Routledge, 1998.

Norbert ELIAS, La Société des individus, Fayard, 1991.

Serge GAGNON, "Attractivité touristique et 'sens' géo-anthropologique des territoires", Téoros, vol. 26, n², 2007.

Erving Goffman, Encounters. Two studies in the Sociology of Interaction, Routledge, 1961.

Maurice HaLBWACHS, Morphologie sociale,

Armand Colin, 1970 (I re édition 1938).

Ulf HANNERZ, Explorer la ville, Les Éditions

de Minuit, 1983.

Michel IZARD et Pierre BONTE, Dictionnaire de l'ethnologie et de l'anthropologie, Puf, 1991.

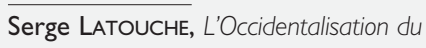
monde. Essai sur la signification, la portée et les limites de l'occidentalisation du monde, La Découverte, 1989.

Naomi Leite et Nelson Graburn,

"L'anthropologie pour étudier le tourisme",

Mondes du tourisme, $\mathrm{n}^{\circ}$ I, 2010.

Emmanuel LÉVINAS, Altérité et transcendance, Puf, 200I.

Franck MICHEL, Désirs d'ailleurs. Essai d'anthropologie des voyages, Presses de l'université Laval, 2004.

Jean-Pierre Mounet et Pierre Chifflet,

"Commercial supply for river water sports", International Review for the Sociology of Sport, vol. 31, n 3, 1996.

Jean-Claude PASSERON, Jean Boutier et Jacques REVEL (dir.), Qu'est-ce qu'une discipline ?, Éditions de l'EHESS, 2006.

Roland ROBERTSON, Globalization. Social Theory and Global Culture, Sage Publications, 1992.

Martin DE LA SOUDIÈRE, Lignes secondaires, Créaphis, 2008.

Jean VIARD, Le Tiers Espace. Essai sur la nature, Méridiens-Klincksieck, 1990.

Michel WIEVIORKA, La Différence culturelle, Balland, 2008.

Raymond WILLIAMS, "Plaisantes perspectives. Invention du paysage et abolition du paysan", Actes de la recherche en sciences sociales, n 17-18, novembre 1977. 